\title{
Fog Computing as a Support for 5G Network
}

\author{
Stojan Kitanov ${ }^{1}$, and Toni Janevski ${ }^{2}$ \\ ${ }^{1}$ Mother Teresa University \\ Faculty of Informatics \\ Skopje, Republic of Macedonia \\ stojan.kitanov@unt.edu.mk \\ ${ }^{2}$ Ss Cyril and Methodius University \\ Faculty of Electrical Engineering and Information Technologies \\ Skopje, Republic of Macedonia \\ tonij@feit.ukim.edu.mk
}

\begin{abstract}
G will enable new future Internet of Services (IoSs) paradigms such as Anything as a Service (AaaS), where devices, terminals, machines, also smart things and robots will become innovative tools that will produce and will use applications, services and data. However, 5G will have to support huge mobile traffic volumes, and will also have to deal with the rapid increase of new and complex applications and services. On the other hand, Fog Computing, which extends Cloud Computing to the edge of the network, with its service orchestration mechanisms offers virtually unlimited dynamic resources for computation, storage and service provision, that will overcome the constraints of smart mobile devices. 5G in the fog computing environment will create opportunities for companies to deploy many new real-time services that cannot be delivered over current mobile and wireless networks. This paper evaluates Fog Computing as a support mechanism for 5G Network in terms of latency, throughput, and energy efficiency.
\end{abstract}

Keywords: 5G, Cloud Computing, Fog Computing, Fog Networking, Mobile Cloud Computing.

\section{Introduction}

The next major phase of mobile telecommunications standards is the Fifth Generation or 5G [1], [2]. 5G will enable the future Internet of Services (IoSs) paradigms such as Anything as a Service (AaaS), where devices, terminals, machines, and also smart things and robots will become innovative tools that will produce and use applications, services, and data [3].

However, future Internet will exacerbate the need for improved QoS/QoE, supported by services that are orchestrated on-demand and are capable of adapt at runtime, depending on the contextual conditions, to allow reduced latency, high mobility, high 
scalability, and real time execution. The emerging wave of Internet of Things (IoTs) would require seamless mobility support and geo-distribution in addition to location awareness and low latency. These demands can only be partially fulfilled by existing cloud computing solutions [4].

A new paradigm called Fog Computing, or Fog Networking has emerged to meet these requirements [5]. Fog extends cloud computing and services to the edge of the network. It provides data, computing, storage, and application services to end-users that can be hosted at the network edge or even end devices such as set-top-boxes or access points. The main features of Fog are its proximity to end-users, its dense geographical distribution, and its support for mobility [6].

By implementing fog computing in 5G network, end-to-end latency will be significantly reduced to less than few milliseconds, and it will improve the service quality perceived by mobile users. This high data speed together with the expected pervasive coverage of reliable networks, will create opportunities for companies to deploy many new real-time services that cannot be delivered over current mobile and wireless networks.

The move from Cloud to Fog in 5G brings out several key challenges, including the need for supporting the on-demand orchestration and runtime adaptation of resilient and trustworthy Fog Services. This is essential for the success of the future Internet of Everything (IoE), which is a clear evolution of the IoT [7].

This paper evaluates Fog Computing as a support for $5 \mathrm{G}$ Network in terms of latency, throughput, and energy efficiency. It is organized as follows. Section 2 provides an overview of 5G services and applications. Section 3 compares $4 \mathrm{G}$ and $5 \mathrm{G}$ requirements. Section 4 considers Fog Computing environment for 5G network. Section 5 performs evaluation of fog computing mechanisms in 5G network in terms of latency, throughput, and energy efficiency. Finally, Section 6 concludes the paper and provides future work research directions.

\section{5G Services and Applications}

$5 \mathrm{G}$ will be a multi-layered heterogeneous network that will consist of existing $2 \mathrm{G}, 3 \mathrm{G}$, 4G and future Radio Access Technologies (RATs). It may also converge many other radio technologies like Mobile Satellite System (MSS), Digital Video Broadcasting (DVB), Wireless Local Access Network (WLAN), Wireless Personal Access network (WPAN), Worldwide Interoperability for Microwave Access network (WiMAX), etc., with multi-tiers coverage by macro, pico, femto, relay and other types of small cells. 5G will support a wide range of applications and services to satisfy the requirements of the information society by the year 2020 and beyond [1], [8].

$5 \mathrm{G}$ network would support a large number of connected devices and flexible air interfaces, different interworking technologies that are energy efficient, and will possess always on-line capabilities [9]. This requires not only upgrade of existing systems, but also innovation of new protocols and new access technologies altogether.

As it is shown in Fig. 1, there are three possible migration paths to 5G network [10], [11]: 
- a step-by-step evolutionary path focusing on further enhancements of existing technologies;

- a revolutionary path using a brand new innovative technologies; or

- a symbiotic integration and convergence of existing or new technologies such as communication, information systems and electronics, multi radio access technologies, computing techniques, device-to-device communications, bands, links, layers, services, multiplexing, etc.

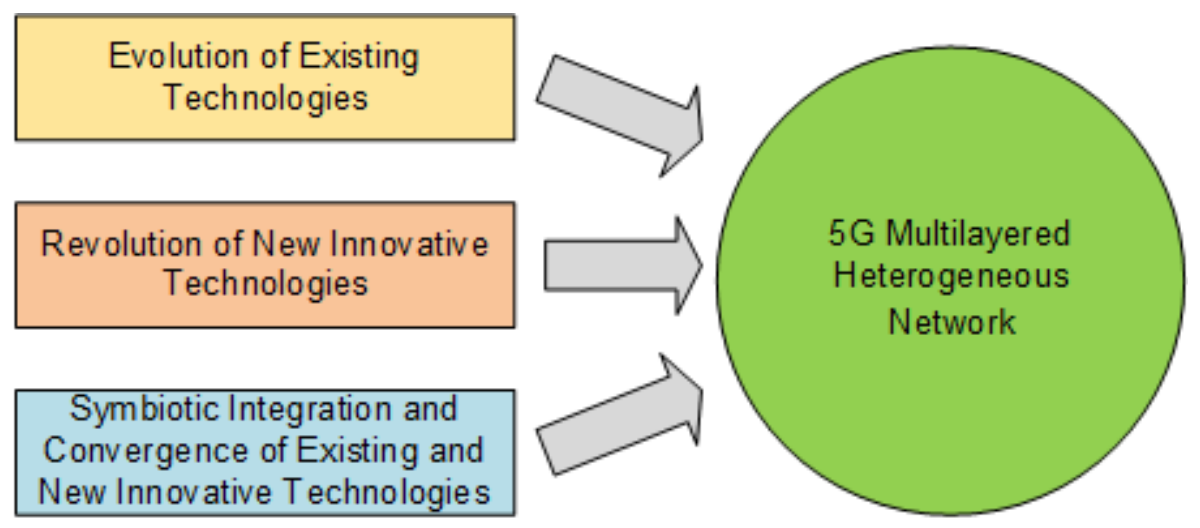

Fig. 1. Technology Routes to 5G

Compared to the previous generations of mobile and wireless networks the development in 5G is focused in the following service types: data, connectivity and user experience (see Table 1 below). 5G will have user-centric approach, where telecom operators will invest in developing new applications that will provide ubiquitous, pervasive, seamless, continual and versatile mobile experience to the end-user [1]. The applications will become more personalized, and more context-aware and that will recognize user identity, user location, and user preferences [12].

Table 1. Service Types from $1 \mathrm{G}$ to $5 \mathrm{G}$

\begin{tabular}{ll}
\hline Network Generation & Service Type \\
\hline $1 \mathrm{G}$ & Analog Voice Communication \\
$2 \mathrm{G}$ & Digital Voice Communication and SMS \\
$3 \mathrm{G}$ & Multimedia \\
$4 \mathrm{G}$ & Mobile Internet \\
$5 \mathrm{G}$ & Data, Connectivity and User Experience \\
\hline
\end{tabular}

Therefore, context-rich support services such as context extraction service, recommendation service and group privacy service should be supported in 5G. Particularly important is the context extraction service that performs data mining analysis of mobile data combined with other forms of data such as social networking data, and sensor network data in order to extract contextual clues relevant to the user. 
Data mining services should be able to scale and analyze large group of people and large quantities of data (big data) in order to extract collective trends among the population of users in real time. Additionally, recommendation services based on collective group context rather than individual context need to be created and scaled. By using these clues, a layer of recommendation services can be built that creates output which is adjusted to a user, or set of users with those contextual characteristics [12].

\section{A Comparison of between 4G and 5G Requirements}

In order to fulfill the demands $5 \mathrm{G}$ systems should deliver a higher order of magnitude cell capacities and per-user data rate compared to $4 \mathrm{G}$. $5 \mathrm{G}$ will have to support huge mobile traffic volumes in the order of multiples of gigabits per second [10]. It will be a set of telecommunication technologies and services that support a 1000 times more data capacity than today, and should provide ultra-low latency response of less than few milliseconds. It will also have to deal with a rapid increase of new and complex applications and services, many of which are unknown today.

The network should provide a capacity of $50 \mathrm{Gbps}$ per cell, and guarantee anywhere more than 1 Gbps per user through super dense networking, regardless of the user location, including the cell edge. The cell spectral efficiency would be increased by 3 to 5 times compared to $4 \mathrm{G}$, and the latency response in control plane would be reduced to one half, i.e. to $50 \mathrm{~ms}$. In addition, it would support ultralow latency response of $1 \mathrm{~ms}$ in data plane, which is equal to one tenth of 4G network [9], [10], [11], [13].

In order to enable the forthcoming Internet of Everything (IoE) [7], 5G should provide 4A (Anytime, Anywhere, Anyone, Anything) massive and simultaneous connectivity that will accommodate one million different mobile devices per unit square kilometer. It would have flexible and intelligent network architecture with software based structure capable to analyze data in real time and would provide intelligent and personalized services [8].

5G should provide reliable secure operation with more than $99 \%$ network availability. There will be possibility for self-healing reconfiguration and self-optimization. The battery life of the mobile devices will be increased to 10 times. Finally, 5G will have lower cost for infrastructure and devices and will be 50 to 100 times more efficient in terms of energy usage per bit, compared to the legacy systems [9].

Two key traffic models should be considered in 5G. These are high-speed video flow from the server to the subscriber and massive Machine-to-Machine (M2M), or Deviceto-Device (D2D) communications [13].

The QoS management mechanisms in 5G networks should provide video and VoIP traffic prioritization towards web-search traffic and other applications tolerant to quality [13]. Some of the QoS parameters are Packet Delay Budget (PDB), or maximum packet delay and Packet Loss Ratio (PLR) that have much lower values to the order of magnitude, than 4G. A comparison between 4G and 5G networks is given in Table 2 . 
Table 2. A Comparison between $4 \mathrm{G}$ and $5 \mathrm{G}$ Networks

\begin{tabular}{|c|c|c|}
\hline Parameter & $4 \mathrm{G}$ & $5 \mathrm{G}$ \\
\hline Air Link User Plane Latency & $10 \mathrm{~ms}$ & $1 \mathrm{~ms}$ \\
\hline Air Link Control Plane Latency & $100 \mathrm{~ms}$ & $50 \mathrm{~ms}$ \\
\hline $\begin{array}{l}\text { Simultaneous Connection Density } \\
\text { per unit } \mathrm{km}^{2}\end{array}$ & $10^{5}$ & $10^{6}$ \\
\hline Mobility & $300 \mathrm{~km} / \mathrm{h}$ & $500 \mathrm{~km} / \mathrm{h}$ \\
\hline Uplink Cell Spectral Efficiency & $1.8 \mathrm{bps} / \mathrm{Hz}$ & $5 \mathrm{bps} / \mathrm{Hz}$ \\
\hline Downlink Cell Spectral Efficiency & $2.6 \mathrm{bps} / \mathrm{Hz}$ & $10 \mathrm{bps} / \mathrm{Hz}$ \\
\hline $\begin{array}{l}\text { Peak Throughput (Downlink) per } \\
\text { Connection }\end{array}$ & $100 \mathrm{Mbps}$ to $1 \mathrm{Gbps}$ & $10 \mathrm{Gbps}$ to $50 \mathrm{Gbps}$ \\
\hline Cell Edge Data Rate & $1 \mathrm{Mbps}$ & 1 Gbps \\
\hline Cost Efficiency & 10 times & 100 times \\
\hline PDB without quality assurance & 100 to $300 \mathrm{~ms}$ & undetermined \\
\hline PDB with guaranteed quality & 50 to $300 \mathrm{~ms}$ & $1 \mathrm{~ms}$ \\
\hline PLR for video broadcasting & $10^{-8}(4 \mathrm{k}$ UHD $)$ & $10^{-9}(8 \mathrm{k}$ UHD $)$ \\
\hline $\begin{array}{l}\text { PLR for M2M services } \\
\text { (without quality assurance) }\end{array}$ & $10^{-3}$ & $10^{-4}$ \\
\hline $\begin{array}{l}\text { PLR for M2M services } \\
\text { (with guaranteed quality) }\end{array}$ & $10^{-6}$ & $10^{-7}$ \\
\hline
\end{tabular}

\section{Fog Computing for $5 \mathrm{G}$ Networks}

The future IoE will become the linkage between extremely complex networked organizations (e.g. telecoms, transportation, financial, health and government services, commodities, etc.), that will provide the basic ICT infrastructure that supports the business processes and the activities of the whole society in general [7]. Frequently, these processes and activities will be supported by orchestrated cloud services, where a number of services work together to achieve a business objective [4].

Although mobile cloud computing is a promising solution for $5 \mathrm{G}$ to cope with the future Internet, still it cannot deal with all future Internet services and applications. This 
is because the future Internet will exacerbate the need for improved QoS/QoE, supported by services that are orchestrated on-demand and that are capable of adapt at runtime, depending on the contextual conditions, to allow reduced latency, high mobility, high scalability, and real time execution. The emerging wave of Internet of Things (IoTs) would require seamless mobility support and geo-distribution in addition to location awareness and low latency. These demands can only be partially fulfilled by existing cloud computing solutions. In addition, cloud computing security mechanisms such as sophisticated access control and encryption have not been able to prevent unauthorized and illegitimate access to data.

Recently a new paradigm called Fog Computing, or briefly Fog has emerged to meet these requirements [5]. Fog Computing extends cloud computing and services to the edge of the network. Fog will combine the study of mobile communications, microclouds, distributed systems, and consumer big data. It is a scenario where a huge number of heterogeneous (wireless and sometimes autonomous) ubiquitous and decentralized devices communicate and potentially cooperate among them and with the network to perform storage and processing tasks without the intervention of third parties [6]. These tasks support basic network functions or new services and applications that run in a sand-boxed environment. Users leasing part of their devices to host these services get incentives for doing so. The distinguishing Fog characteristics are its proximity to endusers, its dense geographical distribution, and its support for mobility. Therefore, Fog paradigm is well positioned for real time big data analytics. Services are hosted at the network edge or even end devices such as set-top-boxes, or access points [14]. By doing so, fog reduces service latency, and improves QoS, resulting in superior user-experience. It supports emerging IoE applications that demand realtime/predictable latency (industrial automation, transportation, networks of sensors and actuators).

The existence of Fog would be enabled by the emerging trends on technology usage patterns on the one side, and the advances on enabling technologies on the other side. Cloud and Fog Computing are mutually beneficial. Certain functions are naturally more advantageous to carry out in Fog while others in Cloud. A comparison between Fog Computing and Cloud Computing is given in [15], which is summarized in Table 3.

Below are given some example cases where fog computing can be applied in 5G.

Case 1: Crowd-sensing the states in $5 G$ base station. Through a collection of $5 \mathrm{G}$ end user client devices may be able to infer the states of a $5 \mathrm{G}$ base station such as the number of Resource Blocks used, by using a combination of passive received signal strength measurement (e.g., RSRQ), active probing (e.g., packet train), application throughput correlation and historical data mining [16].

Case 2: Over The Top (OTT) network provisioning and content management. The traditional approach to innovate in the networks is to introduce another box inside the network. The fog directly leverages the "things" and phones instead, and removes the dependence on boxes-in-the-network altogether. By using end user client devices for the tasks such as Universal Resource Locator (URL) wrapping, content tagging, location tracking, behavior monitoring, network services can be innovated much faster [17].

Case 3: Network selection in a heterogeneous environment. The coexistence of heterogeneous networks (e.g., LTE, femto, WiFi) is a key feature in 5G. Instead of network operator control, 5G will have a user-centric approach where each client can observe its local conditions and make decision on which network to join [1]. Through 
randomization and hysteresis, such local actions may emerge globally to converge to a desirable configuration [18].

Case 3: Borrowing bandwidth from neighbors in $M 2 M$ or D2D communications. When multiple devices are next to each other, one device may request the other devices to share their bandwidth by downloading other parts of the same file and transmitting, via WiFi Direct, client to client, etc. [19].

Case 4: Distributed beam-forming. Fog can also be applied in the physical layer, by exploiting multi-user MIMO to improve throughput and reliability when a client can communicate with multiple access points, or base stations. For uplink, multi-user beamforming so that the client can send multiple data streams to multiple Access Points (Aps), or Base Stations (BSs) simultaneously. For downlink, interference nulling can be used in order the client to be able to decode parallel packets from multiple APs or BSs. These can be done entirely on the client side [20].

Table 3. A Comparison between Cloud and Fog Computing

\begin{tabular}{|c|c|c|}
\hline & Cloud Computing & Fog Computing \\
\hline Target Type & General Internet Users & Mobile Users \\
\hline Service Type & $\begin{array}{l}\text { Global Information } \\
\text { Collected from Worldwide }\end{array}$ & $\begin{array}{l}\text { Limited localized information } \\
\text { services related to specific } \\
\text { deployment locations }\end{array}$ \\
\hline Service Location & Within the Internet & At the edge of the local network \\
\hline $\begin{array}{l}\text { Distance between } \\
\text { client and server }\end{array}$ & Multiple hops & Single hop \\
\hline $\begin{array}{l}\text { Number of server } \\
\text { nodes }\end{array}$ & Few & Very large \\
\hline Latency & High & Low \\
\hline Delay jitter & High & Very low \\
\hline Geo-distribution & Centralized & Distributed \\
\hline Security & Undefined & Can be defined \\
\hline Hardware & $\begin{array}{l}\text { Ample and scalable } \\
\text { storage, } \\
\text { processing and computing } \\
\text { power }\end{array}$ & $\begin{array}{l}\text { Limited storage, processing and } \\
\text { computing power, wireless } \\
\text { interface }\end{array}$ \\
\hline Deployment & $\begin{array}{l}\text { Centralized and maintained } \\
\text { by OTT service providers }\end{array}$ & $\begin{array}{l}\text { Distributed in regional areas and } \\
\text { maintained by local businesses }\end{array}$ \\
\hline
\end{tabular}




\section{Evaluation of Fog Computing for 5G Network}

The performances of fog computing service orchestration environment in $5 \mathrm{G}$ can be explored in many ways such as the Round Trip Time (RTT) latency, throughput, and energy consumption. The following scenario will be used. There is a region in which are located a group of $\mathrm{N}$ smart user devices, which are simultaneously covered by several different RANs. Each RAN is connected to several clouds, which can be in the same, or different region with the RANs. First five clouds are in the same region with the RANs, and the other 5 clouds are in a different region with the RANs. The user devices are assumed to be same smart capabilities capable, and are located on a different distance from the RANs. The smart user devices can be simultaneously served by the RANs and the clouds. The simulation results are based on referent average values for the peak data and the RTT latency of 3G, 4G and 5G Network RAN, that can be found in [21], [22], [23], [24] and are summarized in Table 4. It is assumed that 5G RAN will have 90\% improvement in energy per bit over 4G [13].

Table 4. Throughput, Latency and Energy Efficiency for 3G, 4G and 5G RAN networks

\begin{tabular}{llll}
\hline Parameter & $3 \mathrm{G}$ & $4 \mathrm{G}$ & $5 \mathrm{G}$ \\
\hline Latency [ms] & 70 & 20 & 5 \\
Peak Data Rate [Gbps] & 0.021 & 3 & 40 \\
$\begin{array}{l}\text { Energy per bit for } 10 \\
\text { kB data file [ } 100 \mathrm{~J} / \mathrm{bit}]\end{array}$ & 170 & 17 \\
$\begin{array}{l}\text { Energy per bit for } 10 \\
\mathrm{kB} \text { data file }[\mu \mathrm{J} / \mathrm{bit}]\end{array}$ & 4 & 0.3 & 0.03 \\
\hline
\end{tabular}

\subsection{Latency}

RTT latency is the time it takes for a single data transaction to occur, meaning the time it takes for the packet of data to travel to and from the source to the destination, and back to the source [21], [25]. The RTT latency between the user equipment to any cloud via any RAN is equal to:

$$
R T T=R T T_{R A N}+R T T_{R A N-C L O U D}
$$

where $R T T_{R A N}$ represents RTT RAN latency for 3G, 4G and 5G network, and $R T T_{R A N-C L O U D}$ represents the RTT Latency between the RAN network and the cloud. 


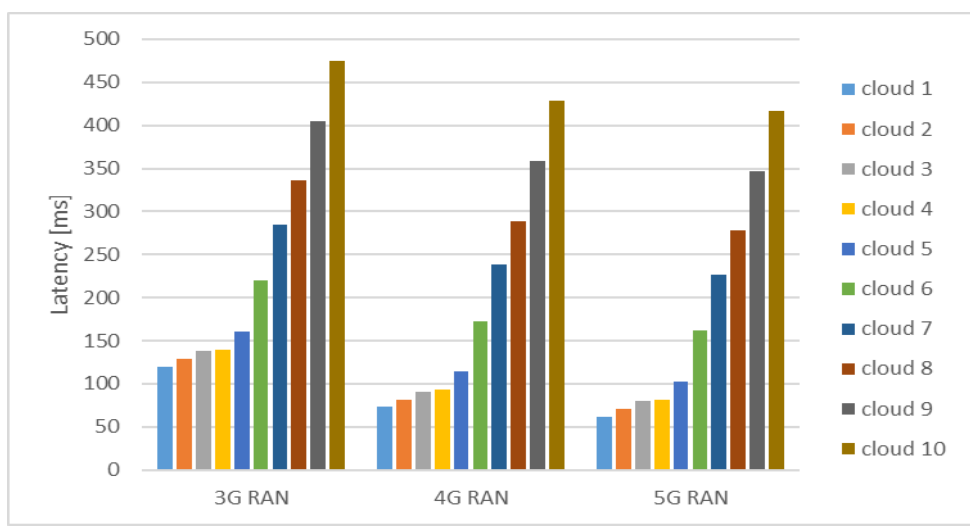

Fig. 2. RTT Latency in the Fog Computing Environment

The average $R T T_{R A N}$ values for $3 \mathrm{G}, 4 \mathrm{G}$ and $5 \mathrm{G}$ are given in Table 4 , and the average values of $R T T_{R A N-C L O U D}$ varies from $50 \mathrm{~ms}$ to $500 \mathrm{~ms}$ depending whether the cloud is in the same or different region with the RAN network. For the simulation purposes randomly generated values were used for $R T T_{R A N-C L O U D}$. Cloud 1 is the least distant from the RANs, and it has the lowest RAN - CLOUD latency, while cloud 10 is the most distant from the RANs, and it has the highest RAN - CLOUD latency.

The simulation results for the RTT latency for different RANs are given in Fig. 2. RTT latency between the user equipment and any cloud is the lowest for 5G RAN, and the highest for 3G RAN, and the user will have preferences to choose the 5G RAN. The RTT latency for any RAN exponentially increases from cloud 1 to cloud 10 , because cloud 1 has the lowest latency to the RANs, and cloud 10 has the highest latency to the RANs.

\subsection{Throughput}

Throughput is the quantity of data that can pass from source to destination in a specific time [25]. The user throughput $T$ can be calculated as a ratio of the peak data rate $R$, and the number of users $N$ :

$$
T=\frac{R}{N}
$$

The peak data rate for $3 \mathrm{G}, 4 \mathrm{G}$ and $5 \mathrm{G}$ RAN is given in Table 4 . The number of the users is varied from 100 to 1000. The user throughput results are shown in Fig. 3. 5G 
offers much higher user throughput than $4 \mathrm{G}$ and $3 \mathrm{G}$. This means that much higher quantity data can pass through $5 \mathrm{G}$ RAN, compared to $4 \mathrm{G}$ and $3 \mathrm{G}$ networks. One important feature in $5 \mathrm{G}$ will be the user equipment to be simultaneously connected to several RANs, i.e. to combine the flows from several RANs, for a single application or service. The combined user throughput is a sum from the user throughputs from the individual RANs. This approach offers the highest user throughput. This is also shown in Fig. 3.

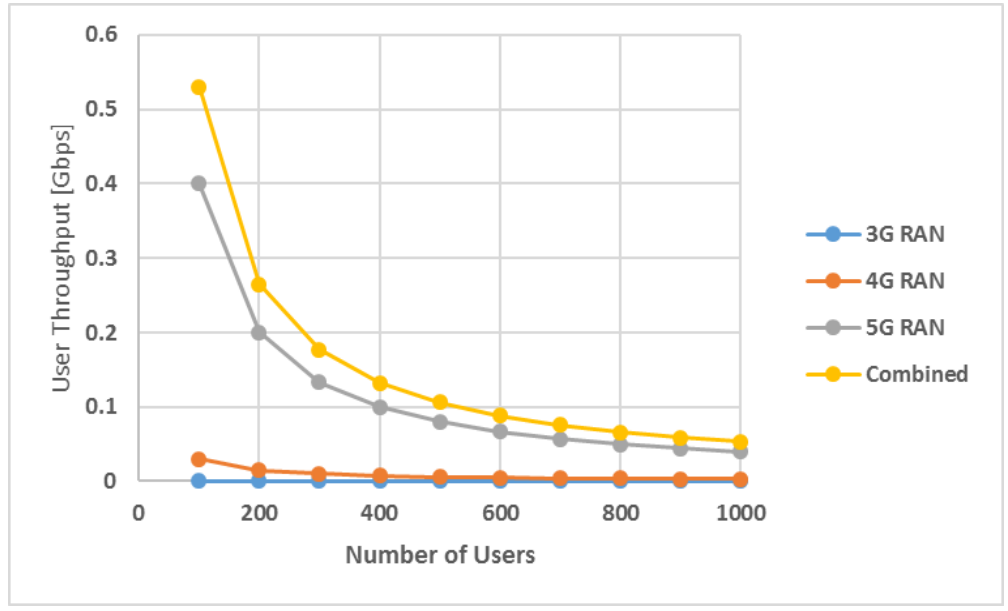

Fig. 3. User Throughput in the Fog Computing Environment

\subsection{Energy Consumption}

The energy consumption per user, that uses fog or cloud computing service is a product of the energy per bit which depends from the RAN type and the size of data file being transferred to the user:

$$
E C=E_{r a n} T
$$

where,

$E_{r a n}$ is the energy per bit that depends from the type of the RAN;

$T$ is the data payload size.

The energy consumption per user is evaluated for different data file size: $10 \mathrm{~KB}$ and $10 \mathrm{MB}$. The most significant impact in the energy consumption will have the RAN type, while the $5 \mathrm{G}$ core impact on the energy efficiency can be treated as a constant, and therefore it can be neglected. 


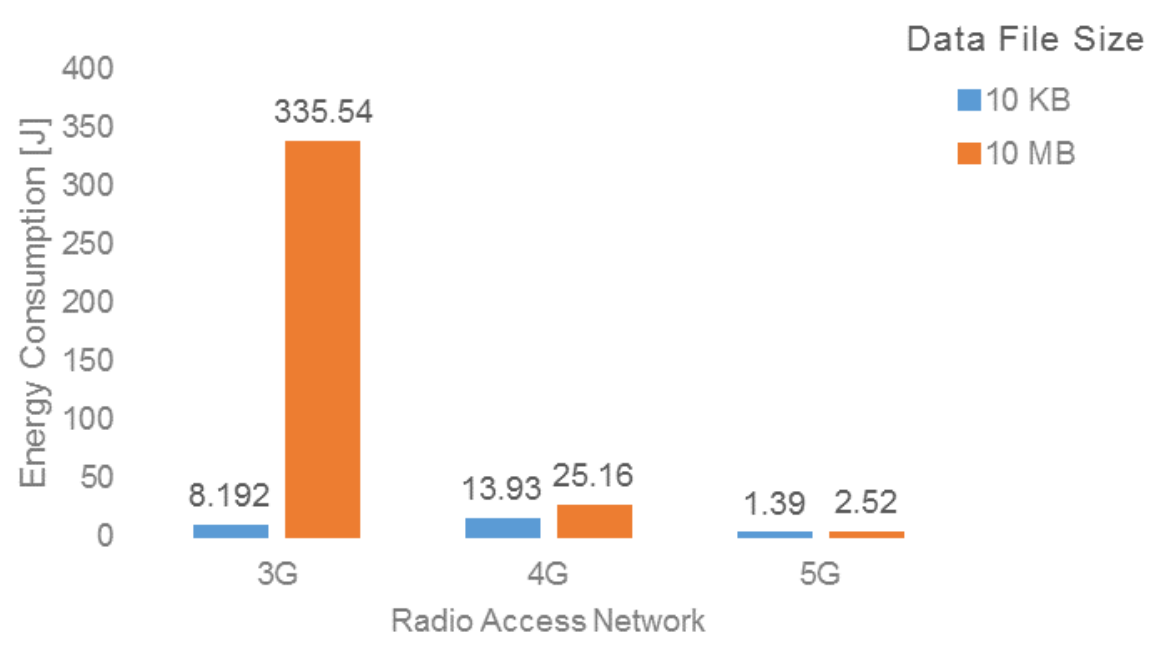

Fig. 4. Energy Consumption in a Fog Computing Environment

The simulation results are provided in Fig. 4. It can be noticed that 3G RAN wastes a lot of energy for the transfer of big data files. 4G RAN provides much better energy efficiency for large data files, compared to 3G RAN. On the other hand, 4G RAN wastes energy for the transfer of small data files, and $3 \mathrm{G}$ RAN demonstrates better performances. Finally, 5G RAN has the best energy efficiency for the transfer independently from the size of data files, that the user is requesting them from the fog or cloud.

In $5 \mathrm{G}$ network where the user device would be served by different RANs, it has to make choice which RAN would be the most suitable for transferring data files. The choice should be made primarily from the size of data files being transferred, throughput, latency, energy consumption, etc. The algorithm for such RAN selection will be our future work direction.

\subsection{Discussion of the Results}

The results clearly demonstrate that $5 \mathrm{G}$ in a fog computing environment will outperform the contemporary communication technologies in terms of latency, user throughput, and energy consumption. In order to satisfy various QoS needs, 5G in the fog can be applied in the following key opportunities: industry, robotics and telepresence, virtual reality, augmented reality, healthcare, road traffic, serious gaming, education and culture, and smart grid.

In particular, the big data analytics that requires real time processing and very often has stringent time requirement can only be carried out in the fog. This is essential for critical usage cases of IoT devices and Tactile Internet that requires $1 \mathrm{~ms}$ end-to-end latency is required in the network in order to provide virtual-reality-type interfaces 
between humans and machines (human-machine interaction and machine-machine interaction) [26].

\section{Conclusion}

5G will use the benefits of the centralized cloud, CRAN and Fog RAN cloud and the distributed Peer-to-Peer mobile cloud among the devices which will create opportunities for companies to deploy many new real-time services that cannot be delivered over current mobile and wireless networks. The cloud in $5 \mathrm{G}$ network will be diffused among the client devices often with mobility too, i.e. the cloud will become fog. More and more virtual network functionality will be executed in a fog computing environment, and that will provide mobiquitous service to the users. This will enable new services paradigms such as Anything as a Service (AaaS) where devices, terminals, machines, and also smart things and robots will become innovative tools that will produce and use applications, services and data. This is particularly important for critical usage cases of IoT devices and Tactile Internet that requires $1 \mathrm{~ms}$ end-to-end latency, and big data analytics that requires real time processing with stringent time requirement that can only be carried out in the fog.

The results about latency, throughput and energy consumption presented in this paper clearly demonstrate the effectiveness and the benefits of implementing fog computing environment in in $5 \mathrm{G}$ network, especially if the flows from different RANs and clouds for a particular application or service requested by the user are combined. The selection of RAN and cloud flows that will be combined and used for a single service or application by the mobile user will depend from the low latency requirements, high throughput requirements, or energy efficiency of the clouds and the RANs. The algorithm for selecting the RAN and cloud flows will be the direction of our future work. In addition, Peer-to-Peer Mobile Cloud will be explored.

\section{References}

1. Janevski, T.: 5G Mobile Phone Concept. In Proceedings of 6th IEEE Consumer Communications and Networking Conference - CCNC 2009, pp. 1-2. (2009)

2. Tudzarov A., Janevski T.: Functional Architecture for 5G Mobile Networks. International Journal of Advanced Science and Technology (IJAST), Vol. 32, pp. 65-78. (2011)

3. Wang X., Chen M., Haleb T.: Cache in the Air: Exploiting Content Caching and Delivery Techniques for 5G Systems. IEEE Communication Magazine, Vol. 52, Issue 2, pp. 131-139. (2014)

4. Zhang, S., Zhang, S., Chen, X., Huo, X.: Cloud Computing Research and Development Trend. In Proceedings of the 2010 Second International Conference on Future Networks (ICFN '10), pp. 93-97. (2010) 
5. Bonomi, F., Milito, R., Zhu, J., Addepalli, S.: Fog Computing and Its Role in the Internet of Things. In Proceedings of the first edition of the MCC workshop on Mobile cloud computing (MCC 2012), Helsinki, Finland, pp. 13-16. (2012)

6. L. M. Vaquero, M., L., Rodero-Merino, L.: Finding your Way in the Fog: Towards a Comprehensive Definition of Fog Computing. ACM SIGCOMM Computer Communication Review, Vol. 44, No. 5, pp. 27-32. (2014)

7. Brech B., Jamison J., Shao L., Wightwick G.: The Interconnecting of Everything. IBM Redbook (2013)

8. Janevski T.: NGN Architectures Protocols and Services. John Wiley \& Sons, UK. (2014)

9. The Road to 5G: Drivers, Applications, Requirements and Technical Development. A GSA (Global mobile Suppliers Association) Executive Report from Ericsson, Huawei and Qualcomm. (2015)

10. SK Telecom Network Technology Research and Development Center 5G White Paper.: SK Telecom's View on 5G Vision, Architecture, Technology, and Service and Spectrum. SK Telecom. (2014)

11. Datang Mobile Wireless Innovation Center 5G White Paper.: Evolution, Convergence and Innovation. Datang Telecom Technology and Industry Group. (2013)

12. Kitanov S., Monteiro E., Janevski T.: 5G and the Fog - Survey of Related Technologies and Research Directions. In Proceedings of the $18^{\text {th }}$ Mediterranean IEEE Electrotechnical Conference MELECON 2016, Limassol, Cyprus. (2016)

13. Tikhvinskiy V., Bochechka G.: Prospects and QoS Requirements in 5G Networks. Journal of Telecommunications and Information Technologies, Vol. 1, No. 1, pp. 23 -26. (2015)

14. Stojmenovic, I., Wen, S.: The Fog Computing Paradigm: Scenarios and Security Issues. Proceedings of the Federated Conference on Computer Science and Information Systems (FedCSIS), ACSIS, Vol. 2, No. 5, pp. 1-8. (2014)

15. Luan H. T., Gao L., Li Z., Sun L. X. Y.: Fog Computing: Focusing on Mobile Users at the Edge. arXiv:1502.01815[cs.NI]. (2015)

16. Chakraborty A., Navda V., Padmanabhan V. N., Ramjee R.: Coordinating cellular background transfers using LoadSense. Proceedings of ACM Mobicom. (2013)

17. Chiang M.: Fog Networking: An Overview on Research Opportunities. white paper. (2015)

18. Aryafar E., Keshavarz-Haddard A., Wang M, Chiang M.: RAT selection games in HetNets. Proceedings of IEEE INFOCOM (2013)

19. Zhang Z., Zhang J., Ying L.: Multimedia streaming in cooperative mobile social networks. Preprint.

20. Du Y., Aryafar E., Camp J., Chiang M.: iBeam: Intelligent client-side multi-user beamforming in wireless networks. Proceedings of IEEE INFOCOM. (2014)

21. Latency - The impact of latency on application performance. Nokia Siemens Networks, White Paper (2009).

22. DMC R\&D Center, Samsung Electronics.: 5G Vision. Samsung Electronics Co., Ltd. White Paper.(2015)

23. Roessler A, Schlienz J., Merkel M. Kottkamp M.: LTE-Advanced (3GPP Rel.12) Technology Introduction. Rhode and Scwharz White Paper, 1MA252_2E. (2014) 
24. Huang J., Qian F., Gerber A., Mao Z. M., Sen S, Spatscheck O.: A Close Examination of Performance and Power Characteristics of 4G LTE Networks. Proceedings of the 10th international conference on Mobile systems, applications, and services (Mobisys 2012), pp. 225-238. (2012)

25. Latency Consideration in LTE Implications to Security Gateway, Stoke Inc. White Paper, Literature No. 130-0029-001.(2014)

26. The Tactile Internet, ITU Technology Watch Report, (2014) 